\title{
The clinical significance of ground glass opacities in lung adenocarcinoma in the era of UICC-TNM classification ver.8
}

\author{
Masayoshi Inoue, Masanori Shimomura \\ Division of Thoracic Surgery, Department of Surgery, Kyoto Prefectural University of Medicine, Kyoto, Japan \\ Correspondence to: Masayoshi Inoue, MD, PhD. Division of Thoracic Surgery, Department of Surgery, Kyoto Prefectural University of Medicine, \\ Kajii-cho 465, Kawaramachi-dori, Hirokoji, Kamigyo-ku, Kyoto 602-8566, Japan. Email: mainoue@koto.kpu-m.ac.jp. \\ Provenance: This is an invited article commissioned by the Section Editor Laura Chiara Guglielmetti (Cantonal Hospital Winterthur, Kantonsspital \\ Winterthur, Switzerland). \\ Comment on: Kim H, Goo JM, Kim YT, et al. Consolidation-to-tumor ratio and tumor disappearance ratio are not independent prognostic factors for \\ the patients with resected lung adenocarcinomas. Lung Cancer 2019;137:123-8.
}

Submitted Nov 18, 2019. Accepted for publication Dec 01, 2019.

doi: $10.21037 /$ jtd.2019.12.58

View this article at: http://dx.doi.org/10.21037/jtd.2019.12.58

I congratulate Dr. Kim on the publication entitled "Consolidation-to-tumor ratio and tumor disappearance ratio are not independent prognostic factors for the patients with resected lung adenocarcinomas". I read with great interest the article by Kim and colleagues (1), which showed that the consolidation-to-tumor ratio (CTR) or the tumor disappearance ratio (TDR) is not an independent prognostic predictor in patients treated with pulmonary resection when using UICC-TNM classification version 8. As Dr. Kim described in the text, CTR or TDR had been historically considered to be one of the prognostic factors in lung cancer patients (Figure 1). Namely, lower CTR or higher TDR indicates a greater proportion of the histological lepidic component, which usually reflects biologically lowgrade malignant behavior in adenocarcinomas (2-5).

Several recent reports, however, showed that CTR is an independent prognostic predictor only in lung cancer smaller than 1 or $2 \mathrm{~cm}$ in diameter $(6,7)$, or that TDR has a significant relationship just with the standard uptake value (SUV) max calculated by positron emission tomographycomputed tomography (PET-CT) scanning (8). It has also been reported that tumor invasiveness or prognosis cannot be predicted by the radiographic appearance (9). We previously reported no lymph node involvement in GGO-dominant lung cancer, though no significant survival superiority was observed on multivariate analyses (10). We also found the usefulness of volume-based CTR with 3-dimensional assessments for accurate pathological staging of lymph

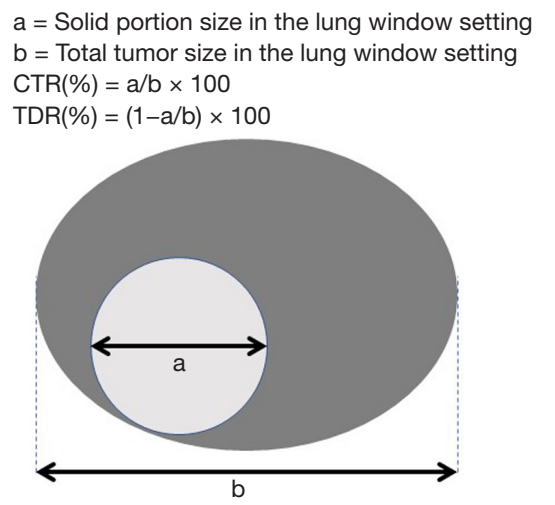

Figure 1 Evaluation of the ground glass area of lung adenocarcinoma in thin-slice computed tomography. CTR, consolidation-to-tumor ratio, TDR, tumor disappearance ratio.

node metastasis in stage I and II adenocarcinoma (11). Tumor diameter is classified in units of $1 \mathrm{~cm}$ in the current UICC-TNM classification version 8, which is well recognized to predict postoperative survival outcomes (12). Thus, the study by Dr. Kim could indicate a change in the clinical significance of the CTR or TDR evaluated using radiographic imaging according to the revised staging or variables for lung cancer. We should meticulously understand the results for GGO appearance calculation, since the lepidic adenocarcinoma component definitively has biological low-grade malignant potential histologically. 


\section{Acknowledgments}

None.

\section{Footnote}

Conflicts of Interest: The authors have no conflicts of interest to declare.

Ethical Statement: The authors are accountable for all aspects of the work in ensuring that questions related to the accuracy or integrity of any part of the work are appropriately investigated and resolved.

\section{References}

1. Kim H, Goo JM, Kim Y'T, et al. Consolidation-to-tumor ratio and tumor disappearance ratio are not independent prognostic factors for the patients with resected lung adenocarcinomas. Lung Cancer 2019;137:123-8.

2. Ohde Y, Nagai K, Yoshida J, et al. The proportion of consolidation to ground-glass opacity on high resolution CT is a good predictor for distinguishing the population of non-invasive peripheral adenocarcinoma. Lung Cancer 2003;42:303-10.

3. Haraguchi N, Satoh H, Kikuchi N, et al. Prognostic value of tumor disappearance rate on computed tomography in advanced-stage lung adenocarcinoma. Clin Lung Cancer 2007;8:327-30.

4. Shimada Y, Yoshida J, Hishida T, et al. Predictive factors of pathologically proven noninvasive tumor characteristics in T1aN0M0 peripheral non-small cell lung cancer. Chest 2012;141:1003-9.

5. Tsurugai Y, Kozuka T, Ishizuka N, et al. Relationship between the consolidation to maximum tumor diameter ratio and outcomes following stereotactic body

Cite this article as: Inoue $M$, Shimomura $M$. The clinical significance of ground glass opacities in lung adenocarcinoma in the era of UICC-TNM classification ver.8. J Thorac Dis 2019;11(12):5680-5681. doi: 10.21037/jtd.2019.12.58 radiotherapy for stage I non-small-cell lung cancer. Lung Cancer 2016;92:47-52.

6. Hattori A, Matsunaga T, Hayashi T, et al. Prognostic Impact of the Findings on Thin-Section Computed Tomography in Patients with Subcentimeter Non-Small Cell Lung Cancer. J Thorac Oncol 2017;12:954-62.

7. Nitadori J, Bograd AJ, Morales EA, et al. Preoperative consolidation-to-tumor ratio and SUVmax stratify the risk of recurrence in patients undergoing limited resection for lung adenocarcinoma $\leq 2 \mathrm{~cm}$. Ann Surg Oncol 2013;20:4282-8.

8. Song SH, Ahn JH, Lee HY, et al. Prognostic impact of nomogram based on whole tumour size, tumour disappearance ratio on CT and SUVmax on PET in lung adenocarcinoma. Eur Radiol 2016;26:1538-46.

9. Ye T, Deng L, Xiang J, et al. Predictors of Pathologic Tumor Invasion and Prognosis for Ground Glass Opacity Featured Lung Adenocarcinoma. Ann Thorac Surg 2018;106:1682-90.

10. Inoue $\mathrm{M}$, Minami $\mathrm{M}$, Shiono $\mathrm{H}$, et al. Clinicopathologic study of resected, peripheral, small-sized, non-small cell lung cancer tumors of $2 \mathrm{~cm}$ or less in diameter: pleural invasion and increase of serum carcinoembryonic antigen level as predictors of nodal involvement. J Thorac Cardiovasc Surg 2006;131:988-93.

11. Shimomura M, Iwasaki M, Ishihara S, et al. VolumeBased Consolidation-to-Tumor Ratio Is a Useful Predictor for Postoperative Upstaging in Stage I and II Lung Adenocarcinomas. Thorac Cardiovasc Surg 2019. [Epub ahead of print].

12. Travis WD, Asamura H, Bankier AA, et al. The IASLC Lung Cancer Staging Project: Proposals for Coding T Categories for Subsolid Nodules and Assessment of Tumor Size in Part-Solid Tumors in the Forthcoming Eighth Edition of the TNM Classification of Lung Cancer. J Thorac Oncol 2016;11:1204-23. 